\title{
Rehabilitation of a Severely Worn Dentition using Fixed and Removable Partial Overdenture Prostheses
}

\author{
${ }^{1}$ Gunjan Pruthi, ${ }^{2}$ Veena Jain, ${ }^{3}$ Hema Agnihotri \\ ${ }^{1}$ Senior Resident, Department of Prosthodontics, All India Institute of Medical Sciences, New Delhi, India \\ ${ }^{2}$ Associate Professor, Department of Prosthodontics, All India Institute of Medical Sciences, New Delhi, India \\ ${ }^{3}$ Senior Resident, Department of Prosthodontics, St George Medical College and Hospital, Mumbai, Maharashtra, India
}

Correspondence: Gunjan Pruthi, Senior Resident, Department of Prosthodontics, All India Institute of Medical Sciences New Delhi, India, e-mail: gunjan_prostho@yahoo.co.in

\section{ABSTRACT}

Planning and executing the restorative rehabilitation of a severely worn dentition is probably one of the most intellectually and technically demanding tasks faced by the restorative dentist. This article describes the rehabilitation of a 55-year-old male patient with severe loss of tooth structure. The maxillary arch was restored using fixed restorations and the mandibular arch using the integration of fixed and removable prosthodontics. The integration of fixed and removable prosthodontics in a partially dentate patient poses a challenge as well as an opportunity for the use of a partial overdenture.

Keywords: Worn dentition, Tooth wear, Fixed removable prosthesis, Telescopic crowns.

\section{INTRODUCTION}

Occlusal wear is most often attributed to attrition, which is defined as the wearing away of one tooth surface by another tooth surface. However, there are several etiological factors that can result in excessive loss of tooth structure. Common factors are enumerated as congenital anomalies, parafunctional occlusal habits, abrasion, erosion or loss of posterior tooth support. ${ }^{1}$ Tooth wear can also be attributed to a coarse diet, to rituals such as filing down teeth and to environmental factors. ${ }^{2}$ Excessive occlusal attrition can result in pulpal pathology, occlusal disharmony, impaired function and esthetic disfigurement. $^{3}$ The reasons for undertaking occlusal rehabilitation may include the restoration of multiple teeth which are missing, worn, broken down or decayed. In certain cases, treatment of temporomandibular disorders (TMD) may also be considered an indication of full mouth rehabilitation, but great caution is advisable in such cases, as the relationship between occlusal rehabilitation and TMD is controversial. ${ }^{4,5}$

Management of the worn dentition is a common problem in the practice of dentistry in terms of both the difficulty and expense of providing a functionally comfortable and cosmetically acceptable treatment of the patient. ${ }^{6}$ Stressinduced grinding habits can seriously compromise mastication and esthetics, and a reduction in the occlusal vertical dimension (OVD) can result in reduction of facial height and initiate angular cheilosis with gradual foreshortening of musculature. ${ }^{7}$ Multiple choices are available for rehabilitation of such patients. Regardless of the clinical reason, the decision of carrying out any treatment should be based upon achieving oral health, function, esthetics and comfort.
The patient in the following case report had presented with severe attrition of all the teeth. The attrition was more severe in the anterior teeth as compared to posterior teeth. There was loss of OVD as determined by various methods, like phonetic evaluation (Pound ${ }^{8}$ and Silverman ${ }^{9}$ ), measurement of interocclusal space (Niswonger). ${ }^{10}$ The anterior lower facial height was found to be collapsed on evaluation of facial appearance. Therefore, according to Pindborg's classification of tooth surface loss (TSL), he was diagnosed to have noncompensated tooth surface loss. According to this classification of TSL, features of non-compensated TSL include tooth surface loss leading to loss of OVD. This is often confined to anterior segments and associated with a lack of posterior occlusion. In such cases, the collapse of anterior facial height necessitates an increase in occlusal vertical dimension to restore the subjects to their presumed, original OVD. ${ }^{11}$

\section{EXAMINATION AND DIAGNOSIS}

A 55-year-old partially-dentate patient with satisfactory general health reported to the Department of Prosthodontics, AIIMS, New Delhi. The patient complained of several missing teeth, excessive wearing away of teeth, reduced chewing efficiency and discomfort due to overclosure. A detailed medical, dental and social history was obtained. Photographs and radiographs were obtained (Fig. 1A). Dental examination revealed partiallydentate maxillary and mandibular arches (Figs 1B and C). Teeth numbers 17, 18, 34, 35, 36 and 46 were missing. All the remaining teeth revealed severe attrition, especially mandibular anterior teeth, which showed severe attrition till gum level. There were multiple faulty restorations and unesthetic 


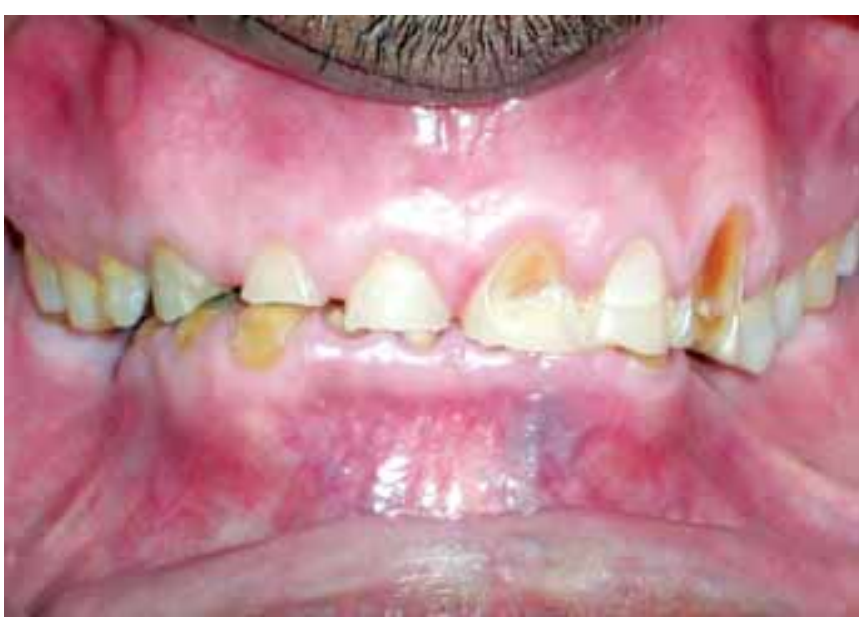

Fig. 1A: Pretreatment frontal view

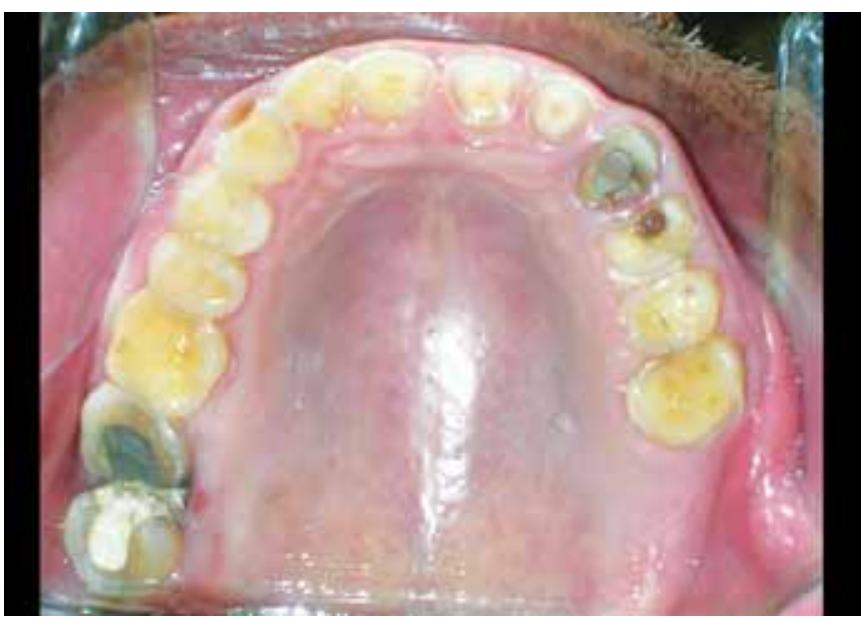

Fig. 1B: Pretreatment maxillary occlusal (mirror) view

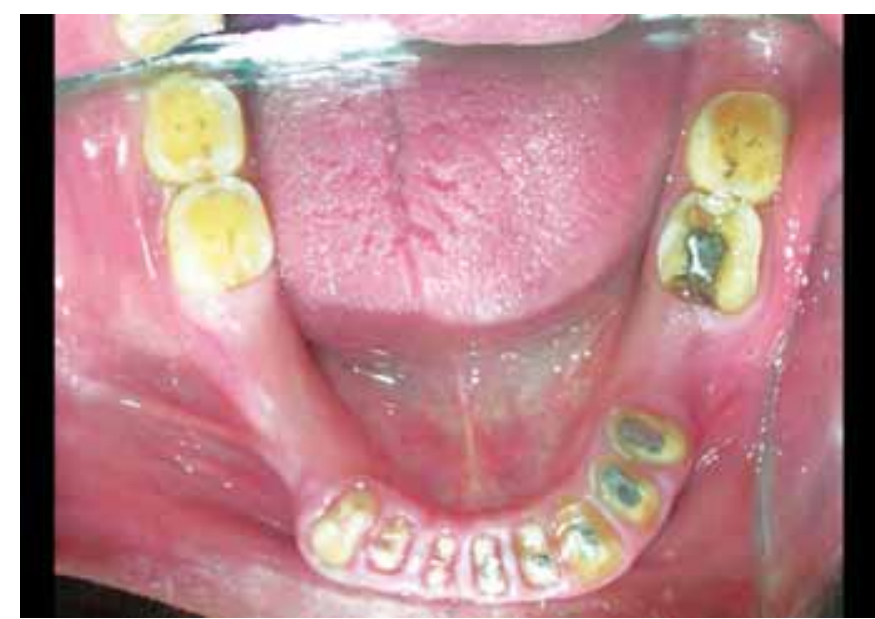

Fig. 1C: Pretreatment mandibular occlusal (mirror) view

appearance of teeth. On TMJ examination, no pain or tenderness was found.

Radiographic examination revealed growth of alveolus in mandibular anterior region leading to insufficient interocclusal space for occlusal rehabilitation. Teeth numbers 13, 27, 31,
32, 33, 41, 42, 43, 44, 45 and 46 were endodontically-treated (Fig. 2). Tooth no. 48 showed furcation involvement. It was periodontally treated to improve its prognosis as abutment. Roots of 31, 32, 41 and 42 had poor prognosis (Fig. 2). Their extraction was planned later on.

On the basis of clinical and radiographic examination, the patient was diagnosed to have excessive wear with loss of occlusal vertical dimension (Turner's category no. 1). ${ }^{3}$ According to the prosthodontic diagnostic index, patient was classified as a PDI class IV with insufficient tooth structure and guarded prognoses for some abutments and requiring re-establishment of the occlusion with a change in the occlusal vertical dimension. ${ }^{12}$

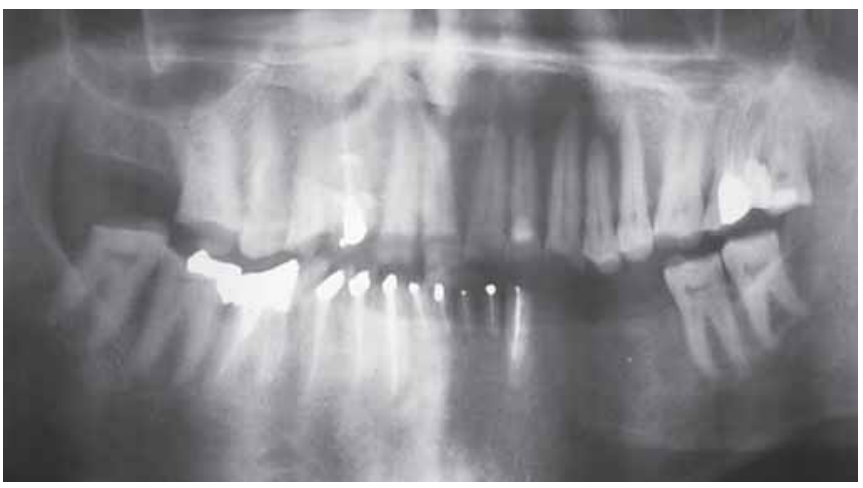

Fig. 2: Pretreatment radiograph

Maxillary and mandibular impressions were made in alginate (Zelgan, Dentsply India Ltd.) to fabricate the diagnostic casts. Occlusal vertical dimension and vertical dimension at rest were assessed by phonetic evaluation (Pound ${ }^{8}$ and Silverman ${ }^{9}$ ), measurement of interocclusal space (Niswonger ${ }^{10}$ ), and evaluation of facial appearance. Maxillary cast was mounted on a semiadjustable articulator (Whip mix Corporation) using a Quick mount earpiece type of facebow whereas the mandibular cast was mounted in centric relation at the established occlusal vertical dimension (Fig. 3).

A maxillary occlusal splint device was fabricated in heatcured clear acrylic resin (Travelon, Dentsply India Ltd.) on another set of mounted casts with uniform occlusal contacts in the posterior quadrants. This was to permit free horizontal movement of mandible to completely deprogram the muscles. Patient was instructed to wear the device for maximum permissible hours per day for 3 months. During this period, various aspects were constantly monitored, such as muscle tenderness, extension of mandibular movements, swallowing, mastication and speech. The occlusal device assisted in deprogramming the muscles of mastication and in assessing the effect of increased occlusal vertical dimension on the TMJ and surrounding musculature. Improvement in speech, esthetics and comfort confirmed the patient's tolerance to the new mandibular position with the restored OVD. On the basis of these observations, it was decided to proceed to the definitive oral rehabilitation at established OVD. 


\section{Diagnostic Wax-Up}

A mandibular mock teeth setting was made to establish the desired esthetics, occlusal plane, tooth contour and positioning for final restoration. Accordingly, a diagnostic wax-up (see Fig. 3) was done on the maxillary cast to establish group function occlusion, which helps to distribute the forces over the greatest possible area on the working side during lateral excursions of the mandible. ${ }^{13}$ Group function occlusion scheme was preferred over canine guided occlusion as canines (13 and 23) of this patient were endodontically treated and were planned for post- and core-retained crowns. So, they were not considered to be good candidates to take the entire load during lateral excursions of the mandible. Roots of 33, 43, 44 and 45 were submerged over which removable prosthesis was planned. The diagnostic wax-up was shown to the patient for his consent.

\section{Definitive Treatment Planning}

All possible treatment modalities and their prognoses were discussed with the patient. A treatment plan was drawn up with the following aims: Restoring the occlusal vertical dimension, improving the esthetics and restoring the masticatory function and comfort of the patient.

The patient did not accept a treatment option utilizing an implant-supported mandibular prosthesis due to finances. Therefore, an overdenture partial prosthesis was planned to restore the integrity of mandibular arch with telescopic crowns on mandibular molars and removable partial denture prosthesis for replacement of the missing mandibular teeth. Metal-ceramic crowns were planned for restoration of teeth in maxillary arch.

\section{Preprosthetic Treatment of Mandibular Arch}

Sufficient space was not available in the anterior region to place teeth due to growth of mandibular alveolar processes. So, alveoloplasty of anterior mandibular ridge along with extraction of teeth numbers 31, 32, 41 and 42 was planned. Root segments of 33, 43, 44 and 45 were preserved for maintenance of alveolar bone and support, improved sensory feedback and masticatory performance. ${ }^{14-17}$

\section{Prosthetic Rehabilitation of Maxillary Arch}

Post and cores were customized for 13, 14 and 23 as minimal coronal tooth structure was left. Tooth preparation of the maxillary teeth was completed followed by gingival retraction (see Fig. 3) and impression was made using polyvinyl siloxane impression material (Reprosil, Dentsply India). Heat cure provisional restorations (DPI heat cure tooth molding powder, Dental products of India, Mumbai) were fabricated according to the diagnostic wax-up and were cemented with temporary cement (Rely X Temp NE, 3M ESPE products). They were adjusted to provide a group function occlusion against the mandibular trial denture (see Fig. 3).

The maxillary master cast was produced in die stone (Kalrock-type IV dental stone, Kalabhai Karson Pvt. Ltd.), and

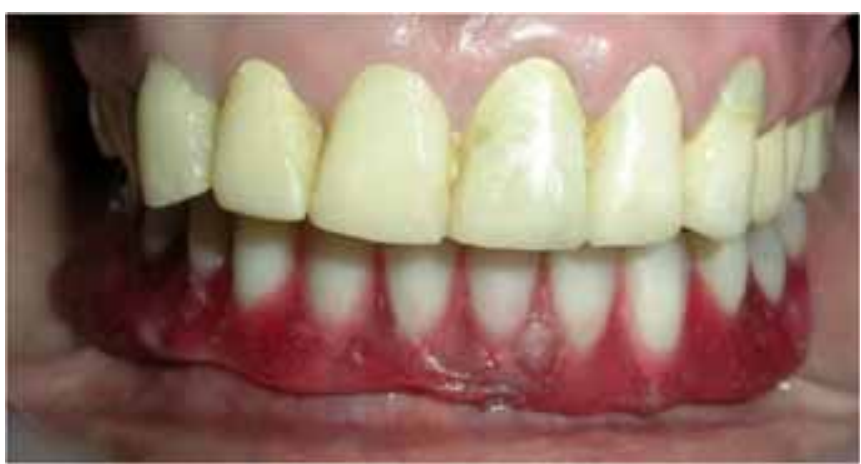

Fig. 3: Maxillary provisional crowns against mandibular trial denture

mounted on semiadjustable articulator (Whipmix co.) against the mandibular master cast (obtained later) after making another face bow record and bite registration. A metal coping try-in was performed for maxillary restorations. Cantilever metalceramic bridge was designed to replace missing 17 taking support from 15 and 16 to provide occlusion against full metal crown on 48.

\section{Rehabilitation of Mandibular Arch}

Teeth numbers 37, 38, 47 and 48 were prepared for telescopic crowns (Fig. 4A). Teeth were prepared with near parallel axial walls. This is because, the more the parallel walls of the coping, the greater the mechanical friction interlocking of the coping with the overlaying crown. ${ }^{18}$ Also, copings contoured with near parallel axial walls of short teeth to a minimal taper provide better resistance form. ${ }^{19}$ Grooves were prepared in buccal and lingual walls of the abutments to achieve more retention. Wax patterns were prepared with cervical shoulder, surveyed to check their parallelism and were cast in chrome cobalt alloy. Cast copings were also surveyed to check the parallelism and determine most suitable path of insertion of the definitive prosthesis. After required adjustments, copings were cemented on molars (Fig 4B). Final impression was made to record complete border extensions of the residual ridge and to record the anatomic details of the metal copings. The mandibular master cast was obtained from this impression and was then duplicated in reversible hydrocolloid duplicating material to obtain the refractory cast for the fabrication of cast metal framework. Refractory cast was articulated against the maxillary diagnostic wax-up, using mandibular trial denture. The definitive waxing for mandibular cast metal framework was performed. Full coverage metal crowns on 37, 38, 47 and 48 were fabricated and the occlusion was adjusted on articulator. The framework was cast in chrome cobalt alloy (Fig 4C). A metal framework try-in was performed to verify intraoral fit, retention and stability. Wax rims were formed on the metal framework and maxillomandibular relations were recorded.

\section{Final Try-in}

A try-in was done before finalizing the maxillary crowns and mandibular denture. Maxillary metal-ceramic crowns were cemented with temporary cement and the mandibular trial 


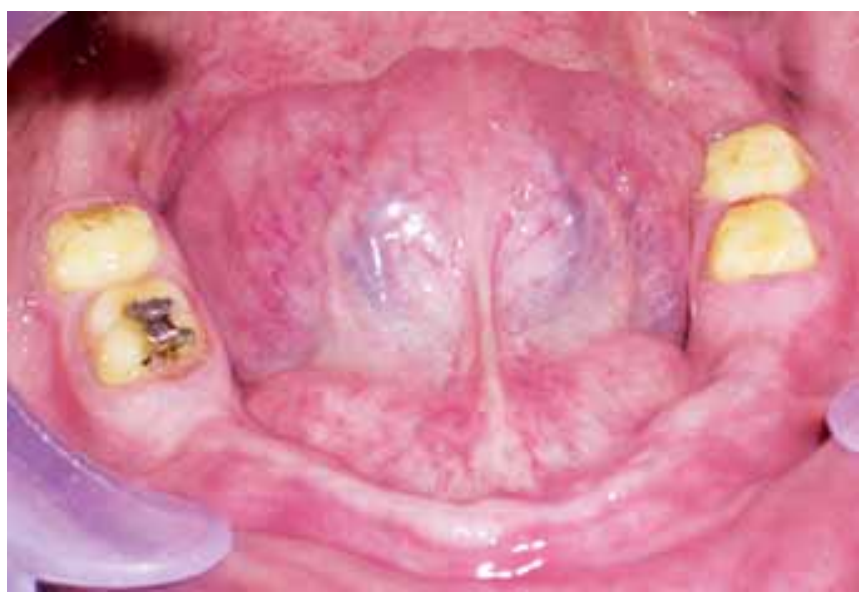

Fig. 4A: Tooth preparation view of mandibular arch

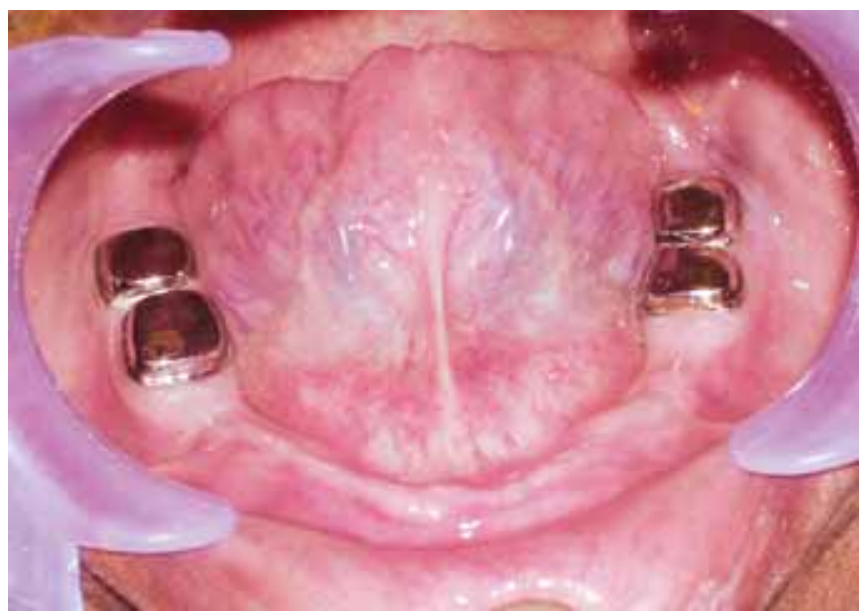

Fig. 4B: Cemented metal copings

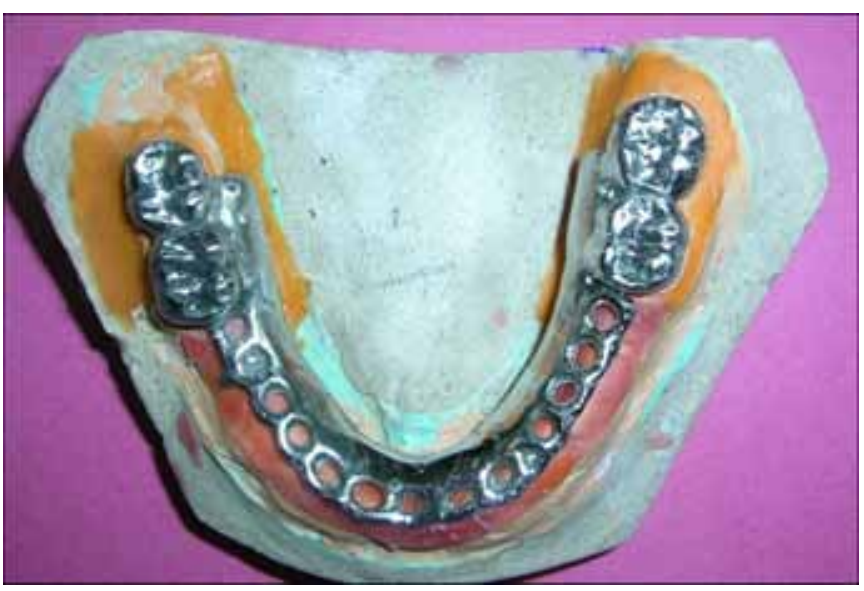

Fig. 4C: Cast metal framework on master cast

denture was seated in the oral cavity to check the occlusion. Modifications were done by adjusting anatomy of maxillary metal-ceramic crowns as it is difficult to grind and polish porcelain teeth. Crowns were adjusted in both centric and eccentric movements and group function occlusion was achieved on both right and left sides (Fig. 5). Metal-ceramic crowns for maxillary arch were finalized and glazed while the mandibular denture was processed. Post-treatment radiograph is shown in Figure 8.

\section{Final Delivery of Prosthesis}

PFM crowns were cemented on maxillary teeth and mandibular denture was delivered to the patient (Figs 6 and 7). Oral hygiene instructions were given to the patient. He was advised to brush teeth twice a day and use dental floss at least once daily to clean the interproximal spaces between the crowns. He was instructed to remove the overdenture during night to give rest to the mandibular mucosa and clean it every morning before wearing it. Patient is being followed up regularly and is satisfied with the performance of the prosthesis. There is no complaint of any pain or discomfort in TMJ region.

\section{DISCUSSION}

The reconstruction of a severely worn dentition is very complex and difficult problem for the dentist. ${ }^{5}$ Excessive wear due to any reason can adversely affect esthetics, function and health of stomatognathic system. So, it becomes important to restore it with any of the suitable treatment options available. A wide range of restorative treatment options are possible with today's materials and techniques. Telescope denture was taken as treatment option for this patient. This design involves an

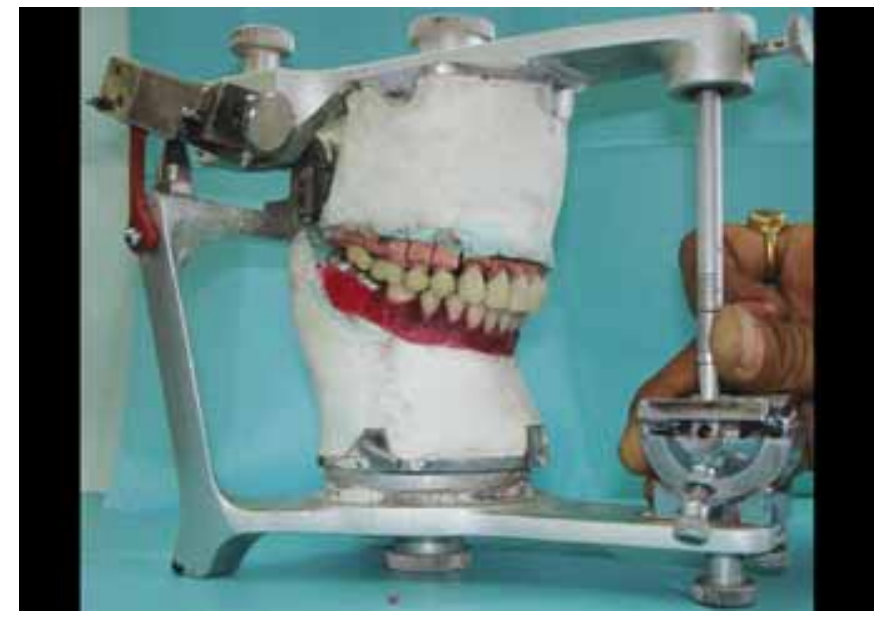

Fig. 5: Maxillary PFM crowns against porcelain teeth of mandibular denture

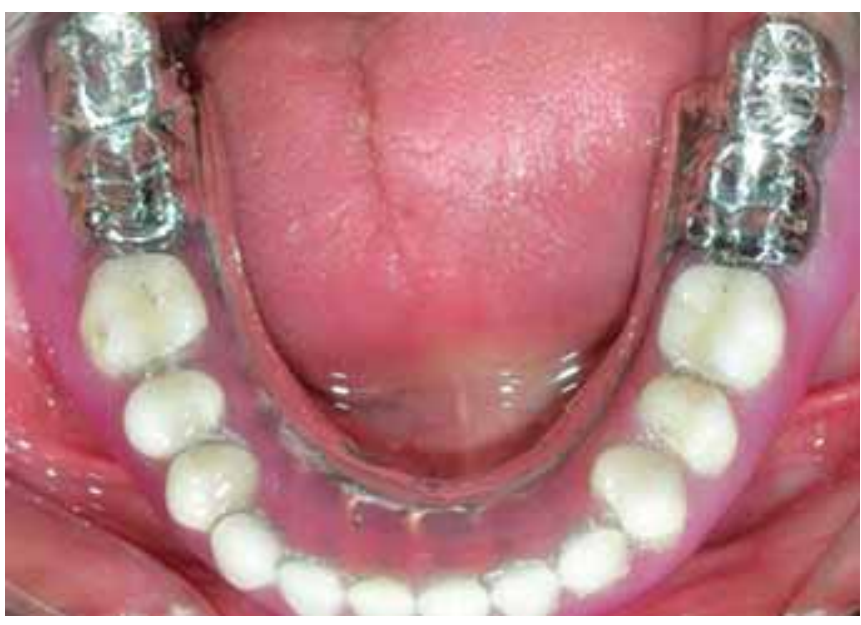

Fig. 6: Mandibular fixed removable prosthesis 


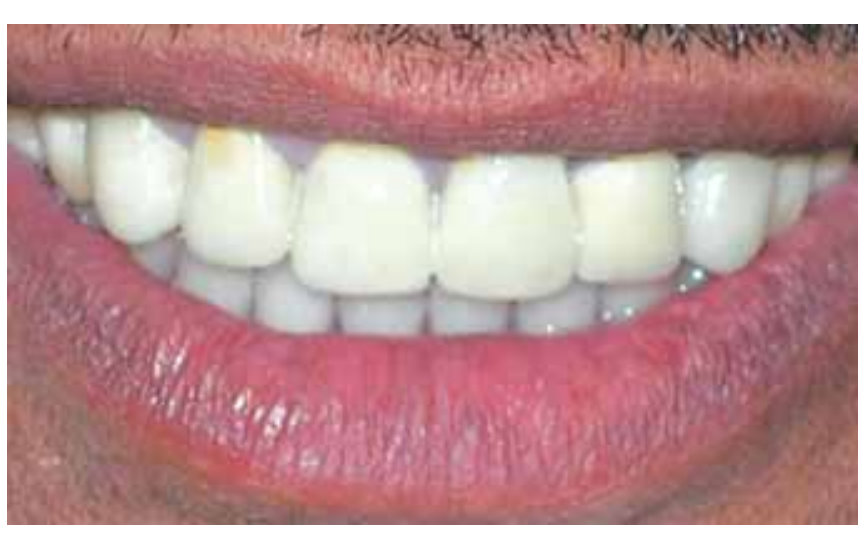

Fig. 7: Post-treatment smiling view

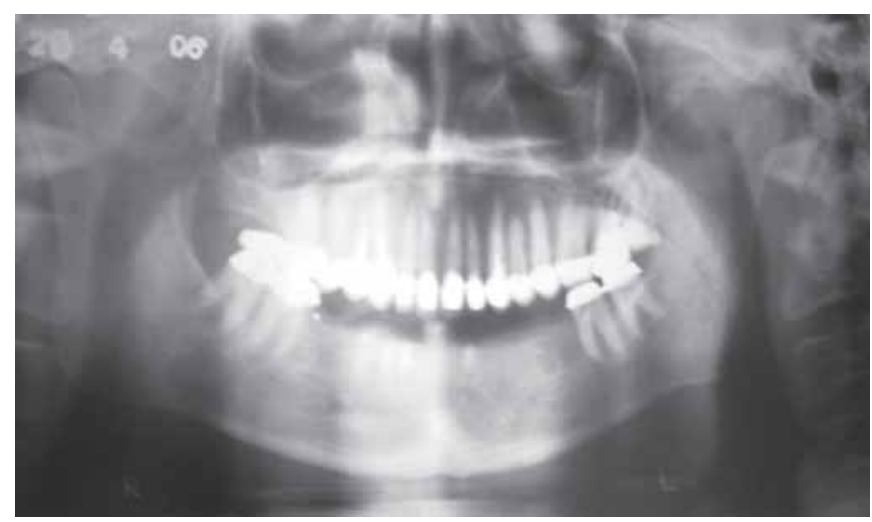

Fig. 8: Post-treatment radiograph

intersurface friction during the insertion and removal of the superstructure as the two parts engage and disengage themselves. ${ }^{18}$ Also, the overdenture maintains teeth as part of the residual ridge. This gives the patient a denture that has far more support than any conventional appliance. Instead of soft, movable mucous membrane, the denture literally sits on teeth "pilings", enabling the denture to withstand a much greater occlusal load without movement. ${ }^{17}$

The telescopic overdenture consists of a primary coping or inner telescope, permanently cemented to the abutment tooth and a congruent secondary or outer crown, rigidly anchored in the removable denture. The secondary crown engages the primary coping to form a telescope retainer unit. The coping protects the abutment tooth from caries and thermal irritations and provides the basic element for retention and stabilization of the outer part. The secondary crown, being an integral part of the removable substructure serves as its anchor with the rest of the dentition. ${ }^{18}$ The other treatment option that was discussed with the patient was of implant placement and implantsupported prostheses. An implant-supported overdenture has definitely a lot of advantages as improved retention, stability and support, prevents bone loss, improved esthetics but the economical constraints of the patient had restrained us to the present treatment option. $^{20}$

So, integration of fixed and removable prosthodontics (overdenture prosthesis) was planned for this patient. The use of overlay prosthesis can be a valuable means of rehabilitating patients with moderate/severe tooth wear. This form of treatment has been advocated by a number of clinicians for patients with forms of tooth wear. ${ }^{7,21,22}$ This approach provides a relatively simple, non-invasive and cost effective way to achieve improvements in appearance and function of the dentition. In this patient, there was collapse of OVD and need for reorganization of occlusion. When reorganizing the occlusion, it is essential to precede restorative procedures with a period of occlusal device therapy to ensure that a stable maxilla-mandibular relationship has been achieved. ${ }^{23}$ An occlusal device was given to the patient to ensure his accommodation to increased OVD. A multidisciplinary approach was taken to rehabilitate the patient's mutilated dentition in an organized way. The successful integration of fixed and removable prosthodontics has resulted in accurately fitting, esthetic and functionally efficient prostheses. Periodic follow-ups and meticulous prostheses maintenance by the patient will hold the key for the ultimate success of these types of rehabilitations.

\section{SUMMARY}

Patient with severe attrition was restored with the help of an overdenture removable type of prosthesis and telescopic fixed restorations. Treatment improved the patient's esthetics and oral function, and established a more favorable plane of occlusion. The patient's social confidence also improved significantly as a result of the dental treatment.

\section{REFERENCES}

1. Smith BGN. Dental erosion, attrition, abrasion. Practitioner 1975;347:55.

2. Holbrook WP, Arnadottir IB. Prevention of tooth wear. Br dent J 2003;195:75-81.

3. Turner KA, Missirlian DM. Restoration of the extremely worn dentition. J Prosthet Dent 1984;52:467-74.

4. Wassell WR, Steele JG. Considerations when planning occlusal rehabilitation: A review of literature. Inter Dent J 1998;48: 571-81.

5. Hammad IA, Nassif NJ, Salameh ZA. Full-mouth rehabilitation following treatment of temporomandibular disorders and teethrelated signs and symptoms. Cranio 2005;23:289-96.

6. Crothers AJR. Tooth wear and facial morphology. J Dent; 20: 333-41.

7. Brown KE. Reconstruction considerations for severe dental attrition. J Prosthet Dent 1980;44:384-88.

8. Pound E. The mandibular movements of speech and their seven related values. J South Calif Dent Soc 1966;34:435.

9. Silverman MM. The speaking method in measuring vertical dimension. J Prosthet Dent 1953;3:193.

10. Niswonger ME. The rest position of the mandible and the centric relation. J Am Dent Assoc 1934;21:1572.

11. Pindborg JJ. Pathology of dental hard tissues. Copenhagen: Munksgaard 1970:300-09.

12. McGarry TJ, Nimmo A, Skiba JF, et al. Classification system for partial edentulism. J Prosthodont 2002;11:181-93.

13. Schuler CH. Considerations of occlusion in fixed partial dentures. Dent Clin North Am 1959;3:175. 
14. Crum RJ, Rooney GE. Alveolar bone loss in overdentures: A 5-year study. J Prosthet Dent 1978; 40(6):610-13.

15. Casey DM, Lauciello FR. A review of the submerged-root concept. J Prosthet Dent 1980;43(2):128-32.

16. Fenton AH. The decade of overdentures: 1970-1980. J Prosthet Dent 1998;79:31-36.

17. DeFranco RL. Overdentures. In: Winkler S (Ed). Essentials of complete denture prosthodontics (2nd ed). Ishiyaku EuroAmerica: Inc. Publishers 2000:384-85.

18. Langer A. Telescope retainers and their clinical application. J Prosthet Dent 1980;44:516-22.
19. Woolsey GD, Matich JA. The effect of axial grooves on the resistance form of cast restorations. J Am Dent Assoc 1978;97: 978-80.

20. Misch CE. Dental implant prosthetics. Elsevier mosby; p. 207.

21. Graser GN, Rogoff GS. Removable partial overdentures for special patients. Dent Clin North Am 1990;34:741-58.

22. Watson RM. The role of removable prostheses and implants in the restoration of the worn dentition. Eur J Prosthodont Restor Dent 1997;5:181-86.

23. Capp NJ. Occlusion and splint therapy. Br Dent J 1999;186: 217-22. 